\title{
Osteomyelitis Caused by Hypervirulent Klebsiella pneumoniae: The First Korean Case With Family Spread
}

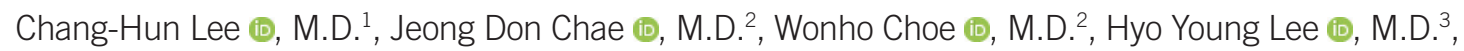 \\ Yong-Hak Sohn (i), M.D. ${ }^{4}$, Chunhwa Ihm (1), M.D. ${ }^{5}$, and Ji Hun Jeong (D), M.D. ${ }^{5}$ \\ Departments of ${ }^{1}$ Orthopaedic Surgery, Hanyang University College of Medicine, ${ }^{2}$ Laboratory Medicine, and ${ }^{3}$ Internal Medicine, Nowon Eulji Medical Center, \\ Eulji University, Seoul, Korea; ${ }^{4}$ Department of Laboratory Medicine, Seegene Medical Foundation, Seoul, Korea; ${ }^{5}$ Department of Laboratory Medicine, \\ Daejeon Eulji Medical Center, Eulji University, Daejeon, Korea
}

\section{Dear Editor,}

Klebsiella pneumoniae is an opportunistic pathogen that causes a wide range of infections. Over the past two decades, a new "hypervirulent" K. pneumoniae (hvKP) strain with hypermucoviscosity, unlike "classic" K. pneumoniae (cKP), has emerged as a clinically significant pathogen causing highly invasive infections, such as liver abscesses, in both healthy and immunocompromised individuals [1, 2]. To the best of our knowledge, only two cases of osteomyelitis due to hvKp have been reported to date $[3,4]$. We present the first case of osteomyelitis due to hvKP spread within a family in Korea. This study was approved by the Institutional Review Board of Nowon Eulji University Hospital, Seoul, Korea. Informed consent was obtained from the patient's husband.

In February 2019, a previously healthy, 60-year-old Korean woman was admitted to a hospital with a three-week history of severe pain in her right thigh. On admission, she was afebrile $\left(37.2^{\circ} \mathrm{C}\right.$ ) but had mild leukocytosis (white blood cell count, $16.9 \times 10^{9} / \mathrm{L}$ ) with elevated C-reactive protein $(3.37 \mathrm{mg} / \mathrm{L}$ ) and procalcitonin $(8.3 \mu \mathrm{g} / \mathrm{L})$ levels. On the second day of admission, magnetic resonance imaging of her right thigh showed in- creased signal intensity in the quadriceps muscle and femur, with bone edema and fluid collection. On the third day of admission, she had fever of $38.9^{\circ} \mathrm{C}$, accompanied by general weakness. After blood and urine cultures were obtained, empirical antibiotic therapy with intravenous tazobactam/piperacillin and vancomycin was initiated. Systemic inflammatory response syndrome (SIRS) was confirmed with leukocytosis (white blood cell count, $30.9 \times 10^{9} / \mathrm{L}$; segmented neutrophils, $89.0 \%$ ), and her respiration rate was 43 breaths/minute. She underwent urgent surgical debridement, and a small abscess with periosteal thickening due to osteomyelitis was observed. Her blood pressure (72/41 $\mathrm{mmHg}$ ) and oxygen saturation (87\%) decreased in the evening. The patient did not recover from SIRS and expired on the fifth day of admission. The urine culture result was found to be positive a day after her death, while two blood culture sets and intraoperative pus culture gave positive results two days after her death. The pathogen was identified as hypermucoviscous $K$. pneumoniae through a positive string test [1].

Osteomyelitis in long bones caused by $K$. pneumoniae is rare; the possible mechanism of osteomyelitis appears to be the hematogenous spread of bacteria colonizing the gastrointestinal
Received: April 24, 2020

Revision received: June 4, 2020

Accepted: September 19, 2020

Corresponding author: Jeong Don Chae, M.D. Department of Laboratory Medicine Nowon Eulji Medical Center, Eulji University, 68 Hangeulbiseok-ro, Nowon-gu, Seoul 01830, Korea Tel: +82-2-970-8324, Fax: +82-2-970-8534

E-mail: jdchae@eulji.ac.kr

\section{(c) (1) (8)}

(C) Korean Society for Laboratory Medicine

This is an Open Access article distributed under the terms of the Creative Commons Attribution Non-Commercial License (https://creativecommons.org/licenses/by-nc/4.0) which permits unrestricted non-commercial use, distribution, and reproduction in any medium, provided the original work is properly cited. 
tract [3]. Our investigation into the cause of the invasive $K$. pneumoniae infection in this patient revealed that her husband had been treated for a K. pneumoniae liver abscess three years ago. One month after the patient died, her husband's stool was cultured, and $K$. pneumoniae was isolated from it.

We characterized the antimicrobial susceptibility of two isolates from the patient's blood samples and her husband's stool sample using the VITEK-2 system (BioMérieux, Marcy-I'Étoile,
France) and genetic relatedness using capsular typing and multilocus sequence typing. Both isolates were susceptible to all antibiotics except ampicillin, similar to susceptibility patterns reported in China [5] and the USA [3]. The K. pneumoniae isolates were identified as capsular serotype/genotype $\mathrm{K} 1$ and sequence type 23 (ST23). K. pneumoniae K1 ST23 strains closely associated with liver abscesses were identified in $4.9 \%$ of healthy Koreans in 2007 [6]. The familial spread of K. pneu-
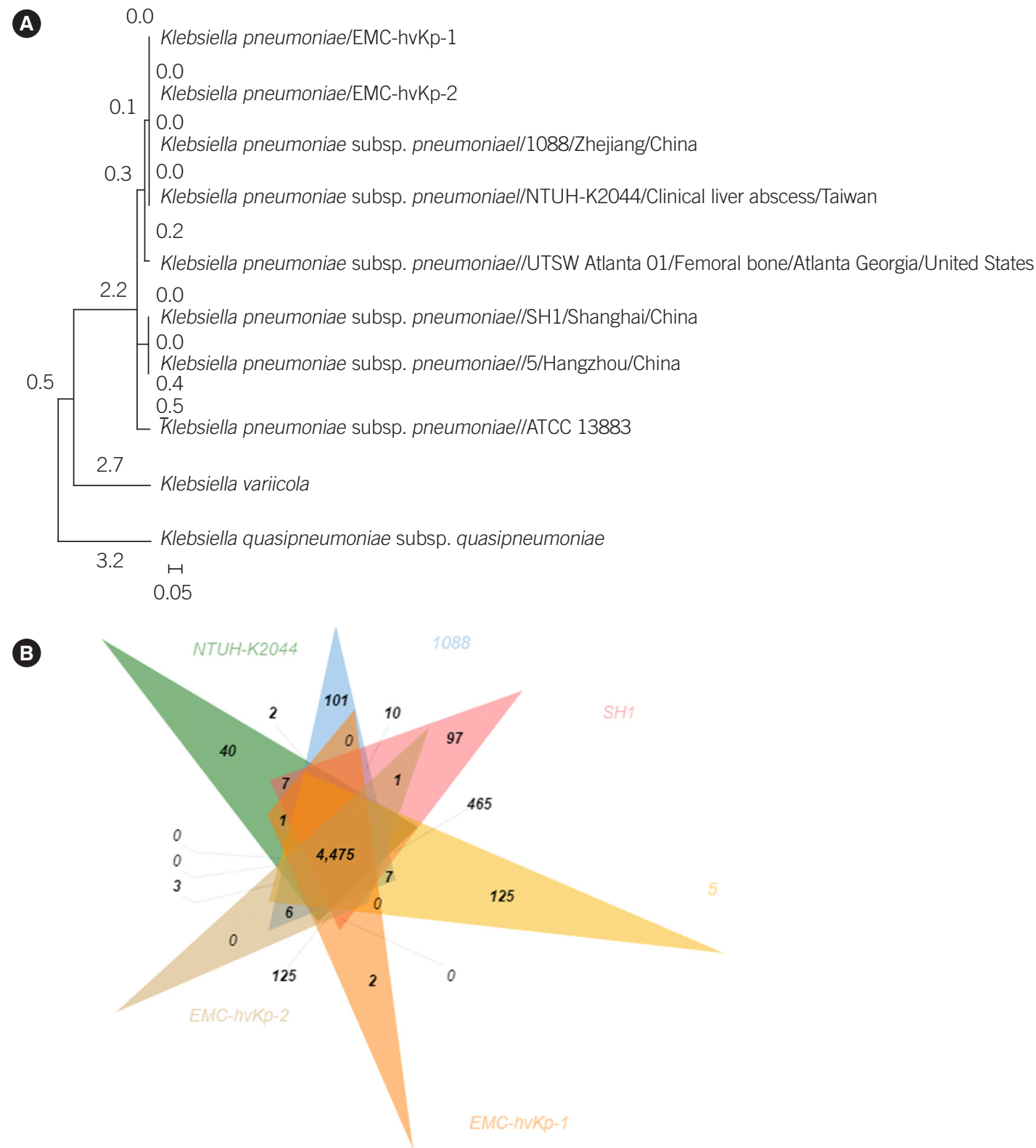

Fig. 1. Characterization of the two Klebsiella pneumoniae isolates from the present case. (A) Phylogenetic tree analysis based on tetra-nucleotide analysis. (B) Venn diagram of the six clinically associated hypervirulent Klebsiella pneumoniae (hvKp) isolates.

Abbreviations: EMC-hvKp-1, blood isolate from the patient; EMC-hvKp-2, stool isolate from the patient's husband; NTUH-K2044, liver abscess isolate from Taiwan; UTSW Atlanta 01, femoral bone isolate from the USA; ATCC 13883, K. pneumoniae type strain; 1088, carbapenem-resistant hvKP strain from China; $\mathrm{SH}-1$, carbapenem-resistant K. pneumoniae strain from China; 5, carbapenem-resistant hvKP strain from China. 
Table 1. Virulence gene analysis of the $K$. pneumoniae strains *

\begin{tabular}{|c|c|c|c|c|c|c|c|c|}
\hline Locus & EMC_hvkp_1 & EMC_hvkp_2 & NTUH-K2044 & UTSW Atlanta 01 & 1088 & ATCC 13883 & SH1 & 5 \\
\hline MLST & 23 & 23 & 23 & 23 & 23 & 3 & 11 & 11 \\
\hline allA & 0 & 0 & 0 & 0 & 0 & & & \\
\hline allB & 0 & 0 & 0 & 0 & 0 & & & \\
\hline allc & 0 & 0 & 0 & 0 & 0 & & & \\
\hline allD & 0 & 0 & 0 & 0 & 0 & & & \\
\hline allR & 0 & 0 & 0 & 0 & 0 & & & \\
\hline alls & 0 & 0 & 0 & 0 & 0 & & & \\
\hline $\operatorname{arcc}$ & 0 & 0 & 0 & 0 & 0 & & & \\
\hline$c \mid b A$ & 0 & 0 & & 0 & 0 & 0 & & \\
\hline \multicolumn{9}{|l|}{$c l b B$} \\
\hline$c \mid b C$ & 0 & 0 & & 0 & 0 & 0 & & \\
\hline$c \mid b D$ & 0 & 0 & & 0 & 0 & 0 & & \\
\hline$c \mid b E$ & 0 & 0 & & 0 & 0 & 0 & & \\
\hline$c l b F$ & 0 & 0 & & & 0 & 0 & & \\
\hline$c \mid b G$ & 0 & 0 & & 0 & 0 & 0 & & \\
\hline$c / b H$ & 0 & 0 & & 0 & 0 & 0 & & \\
\hline$c|b|$ & 0 & 0 & & 0 & 0 & 0 & & \\
\hline$c l b J$ & & & & & 0 & 0 & & \\
\hline clbK & & & & & 0 & & & \\
\hline$c \mid b L$ & 0 & 0 & & 0 & 0 & 0 & & \\
\hline$c l b M$ & 0 & 0 & & 0 & 0 & 0 & & \\
\hline$c l b N$ & 0 & 0 & & 0 & 0 & 0 & & \\
\hline$c / b 0$ & 0 & 0 & & 0 & 0 & 0 & & \\
\hline$c \mid b P$ & 0 & 0 & & 0 & 0 & 0 & & \\
\hline$c \mid b Q$ & 0 & 0 & & 0 & 0 & 0 & & \\
\hline$c \mid b R$ & 0 & 0 & & 0 & 0 & 0 & & \\
\hline$f d r A$ & 0 & 0 & 0 & 0 & 0 & & & \\
\hline fyuA & 0 & 0 & 0 & 0 & 0 & 0 & 0 & \\
\hline$g c l$ & 0 & 0 & 0 & & 0 & & & \\
\hline$g / x K$ & 0 & 0 & 0 & 0 & 0 & & & \\
\hline$g / x R$ & 0 & 0 & 0 & 0 & 0 & & & \\
\hline hyi & 0 & 0 & 0 & 0 & 0 & & & \\
\hline iroB & 0 & 0 & 0 & & 0 & & & \\
\hline iroc & 0 & 0 & 0 & & 0 & & & \\
\hline iroD & 0 & 0 & 0 & & 0 & & 0 & \\
\hline iroN & 0 & 0 & 0 & 0 & 0 & & 0 & \\
\hline \multicolumn{9}{|l|}{ irpl } \\
\hline irp2 & 0 & 0 & 0 & 0 & 0 & 0 & 0 & \\
\hline iucA & 0 & 0 & 0 & & 0 & & 0 & \\
\hline iucB & 0 & 0 & 0 & 0 & 0 & & 0 & \\
\hline iucc & 0 & 0 & 0 & & 0 & & 0 & \\
\hline$i u c D$ & 0 & 0 & 0 & & 0 & & 0 & \\
\hline
\end{tabular}


Lee $\mathrm{CH}$, et al.

Osteomyelitis due to Hypervirulent K. pneumoniae

Table 1. Continued

\begin{tabular}{|c|c|c|c|c|c|c|c|c|}
\hline Locus & EMC_hvkp_1 & EMC_hvkp_2 & NTUH-K2044 & UTSW Atlanta 01 & 1088 & ATCC 13883 & SH1 & 5 \\
\hline MLST & 23 & 23 & 23 & 23 & 23 & 3 & 11 & 11 \\
\hline iutA & 0 & 0 & 0 & & 0 & & 0 & \\
\hline kfuA & 0 & 0 & 0 & 0 & 0 & & & \\
\hline kfuB & 0 & 0 & 0 & 0 & 0 & & & \\
\hline kfuC & 0 & 0 & 0 & 0 & 0 & & & \\
\hline KP1_1364 & 0 & 0 & 0 & 0 & 0 & & & \\
\hline KP1_1371 & 0 & 0 & 0 & & 0 & & & \\
\hline kvgA & & & & & & 0 & & \\
\hline kvgS & & & & & & 0 & & \\
\hline mсеA & 0 & 0 & & 0 & 0 & & & \\
\hline$m c e B$ & 0 & 0 & & 0 & 0 & & & \\
\hline mceC & 0 & 0 & & 0 & 0 & & & \\
\hline mced & 0 & 0 & & 0 & 0 & & & \\
\hline$m c e E$ & 0 & 0 & & 0 & 0 & & & \\
\hline mceG & 0 & 0 & & 0 & 0 & & & \\
\hline mсеH & 0 & 0 & & 0 & 0 & & & \\
\hline mcel & 0 & 0 & & 0 & 0 & & & \\
\hline mceJ & 0 & 0 & & 0 & 0 & & & \\
\hline$m r k A$ & 0 & 0 & 0 & 0 & 0 & 0 & 0 & \\
\hline$m r k B$ & 0 & 0 & 0 & 0 & 0 & 0 & 0 & \\
\hline$m r k C$ & 0 & 0 & 0 & 0 & 0 & 0 & 0 & \\
\hline$m r k D$ & 0 & 0 & 0 & 0 & 0 & 0 & 0 & \\
\hline$m r k F$ & 0 & 0 & 0 & 0 & 0 & 0 & 0 & \\
\hline$m r k H$ & 0 & 0 & 0 & 0 & 0 & 0 & 0 & \\
\hline$m r k l$ & 0 & 0 & 0 & 0 & 0 & 0 & & \\
\hline$m r k J$ & 0 & 0 & 0 & 0 & 0 & 0 & 0 & \\
\hline rmpA & 0 & 0 & 0 & 0 & 0 & 0 & & \\
\hline rmpA2 & 0 & 0 & 0 & & 0 & & 0 & \\
\hline$y b b W$ & 0 & 0 & 0 & 0 & 0 & & & \\
\hline$y b b Y$ & 0 & 0 & 0 & 0 & 0 & & & \\
\hline$y b t A$ & 0 & 0 & 0 & 0 & 0 & 0 & 0 & \\
\hline$y b t E$ & 0 & 0 & 0 & 0 & 0 & 0 & 0 & \\
\hline$y b t P$ & 0 & 0 & 0 & & 0 & 0 & 0 & \\
\hline$y b t Q$ & 0 & 0 & 0 & 0 & 0 & 0 & 0 & \\
\hline ybtS & 0 & 0 & 0 & 0 & 0 & 0 & 0 & \\
\hline$y b t T$ & 0 & 0 & 0 & 0 & 0 & 0 & 0 & \\
\hline ybtU & 0 & 0 & 0 & 0 & 0 & 0 & 0 & \\
\hline$y b t x$ & 0 & 0 & 0 & 0 & 0 & 0 & 0 & \\
\hline$y \mid b E$ & 0 & 0 & 0 & 0 & 0 & & & \\
\hline$y \mid b F$ & 0 & 0 & 0 & 0 & 0 & & & \\
\hline
\end{tabular}

*The heatmaps were generated by aligning the draft genome sequence of each strain to the virulence gene database (https://bigsdb.pasteur.fr/klebsiella/klebsiella.html).

Abbreviations: EMC-hvKp-1, blood isolate from the patient; EMC-hvKp-2, stool isolate from the patient's husband; NTUH-K2044, liver abscess isolate from Taiwan; UTSW Atlanta 01, femoral bone isolate from the USA; ATCC 13883, K. pneumoniae type strain; 1088, carbapenem-resistant hvKP strain from China; $\mathrm{SH}-1$, carbapenem-resistant $K$. pneumoniae strain from China; 5, carbapenem-resistant hvKP strain from China; MLST, multilocus sequence typing. 
moniae $\mathrm{K} 1$ isolates causing liver abscesses was first reported in Japan in 2011 [7]. Most hvKP strains are type K1 or K2 and have remained antibiotic-sensitive.

Whole-genome sequencing using the MiSeq (Illumina Inc., San Diego, CA, USA) platform and comparative genomic analysis of the two K. pneumoniae isolates were conducted by ChunLab, Seoul, Korea. The phylogenetic tree shows that the two isolates in this case are closely related to a liver abscess isolate from Taiwan and a femoral bone isolate from the USA [3, 8] (Fig. 1A); a carbapenem-resistant K. pneumoniae strain and carbapenem-resistant hvKP strains from China are also closely related $[9,10]$. Fig. $1 B$ presents a comparative Venn diagram of six hvKP strains sharing multiple genes $(4,475)$; the two strains in this case are more closely related to each other than they are to the other strains. As seen in this case, a number of virulence factors in hypermucoviscous isolates are associated with a large virulence plasmid, which encodes multiple virulence factors, including rmpA and rmpA2 (Table 1). The capsular genotype (K1 or K2) plays a more significant role than magA and rmpA in determining $K$. pneumoniae virulence in liver abscesses [3].

In conclusion, HvKp can be a member of the gut microbiome, which likely contributes to its dissemination in communities and hospitals. Microbiologists and clinicians should be more aware of the possible severity of infections caused by this strain of $K$. pneumoniae.

\section{ACKNOWLEDGEMENTS}

None.

\section{AUTHOR CONTRIBUTIONS}

CHL and HYL obtained clinical background data of the patient and her husband. YHS and JHJ analyzed the data and wrote the manuscript. JDC, WC, and CHL wrote the manuscript. JDC and WC contributed to manuscript revision. All authors have read and approved the final manuscript.

\section{CONFLICTS OF INTEREST}

None declared.

\section{RESEARCH FUNDING}

None declared.

\section{ORCID}

Chang-Hun Lee

https://orcid.org/0000-0003-4330-7726

Jeong Don Chae https://orcid.org/0000-0002-2654-8299

Wonho Choe

Hyo Young Lee

https://orcid.org/0000-0002-9685-4291

https://orcid.org/0000-0001-7993-3572

Yong-Hak Sohn

Chunhwa Ihm

https://orcid.org/0000-0002-1539-9304

https://orcid.org/0000-0002-8757-3949

Ji Hun Jeong

\section{REFERENCES}

1. Shon AS, Bajwa RPS, Russo TA. Hypervirulent (hypermucoviscous) Klebsiella pneumoniae: a new and dangerous breed. Virulence 2013; 4:107-18.

2. Liu Z, Gu Y, Li X, Liu Y, Ye Y, Guan S, et al. Identification and characterization of NDM-1-producing hypervirulent (hypermucoviscous) Klebsiella pneumoniae in China. Ann Lab Med 2019; 39:167-75.

3. Prokesch BC, TeKippe M, Kim J, Raj P, TeKippe EM, Greenberg DE. Primary osteomyelitis caused by hypervirulent Klebsiella pneumoniae. Lancet Infect Dis 2016;16:e190-5.

4. Sturm E, Tai A, Lin B, Kwong J, Athan E, Howden BP, et al. Bilateral osteomyelitis and liver abscess caused by hypervirulent Klebsiella pneumoniae- a rare clinical manifestation (case report). BMC Infect Dis 2018;18:380

5. Li W, Sun G, Yu Y, Li N, Chen M, Jin R, et al. Increasing occurrence of antimicrobial-resistant hypervirulent (hypermucoviscous) Klebsiella pneumoniae isolates in China. Clin Infect Dis 2014;58:225-32.

6. Chung DR, Lee H, Park MH, Jung SI, Chang HH, Kim YS, et al. Fecal carriage of serotype K1 Klebsiella pneumoniae ST23 strains closely related to liver abscess isolates in Koreans living in Korea. Eur J Clin Microbiol Infect Dis 2012;31:481-6.

7. Harada S, Tateda K, Mitsui H, Hattori Y, Okubo M, Kimura S, et al. Familial spread of a virulent clone of Klebsiella pneumoniae causing primary liver abscess. J Clin Microbiol 2011;49:2354-6.

8. Wu KM, Li LH, Yan JJ, Tsao N, Liao TL, Tsai HC, et al. Genome sequencing and comparative analysis of Klebsiella pneumoniae NTUHK2044, a strain causing liver abscess and meningitis. J Bacteriol 2009; 191:4492-501.

9. Zhang R, Lin D, Chan EW, Gu D, Chen GX, Chen S. Emergence of carbapenem-resistant serotype K1 hypervirulent Klebsiella pneumoniae strains in China. Antimicrob Agents Chemother 2016;60:709-11.

10. Gu D, Dong N, Zheng Z, Lin D, Huang M, Wang L, et al. A fatal outbreak of ST11 carbapenem-resistant hypervirulent Klebsiella pneumoniae in a Chinese hospital: a molecular epidemiological study. Lancet Infect Dis 2018;18:37-46. 